\title{
Prediction of Collective Characteristics for Ion Ensembles in Quadrupole Ion Traps Without Trajectory Simulations
}

\author{
Douglas E. Goeringer \\ Organic and Biological Mass Spectrometry Group, Chemical Sciences Division, Oak Ridge National \\ Laboratory, Oak Ridge, Tennessee, USA
}

\author{
Larry A. Viehland and Daniel M. Danailov \\ Division of Science, Chatham College, Pittsburgh, Pennsylvania, USA
}

\begin{abstract}
Fundamental aspects are presented of a two-temperature moment theory for quadrupole ion traps developed via transformation of the Boltzmann equation. Solutions of the moment equations correspond to changes in the ensemble average for any function of ion velocity, because the Boltzmann equation reflects changes to an ion distribution as a whole. The function of primary interest in this paper is the ion effective temperature and its behavior during ion storage and resonance excitation. Calculations suggest that increases in ion effective temperature during resonance excitation are due primarily to power absorption from the main $\mathrm{RF}$ trapping field rather than from the dipolar excitation signal. The dipolar excitation signal apparently serves mainly to move ions into regions of the ion trap where the RF electric field, and thus ion RF heating, is greater than near the trap center. Both ideal and non-ideal ion trap configurations are accounted for in the moment equations by incorporating parameterized variables $\widetilde{a}$ and $\tilde{q}$, which are modified versions of the commonly used forms for the DC and AC ring voltages, and $\tilde{b}$ and $\tilde{d}$, which are new forms that account for the voltages applied to the endcaps. Besides extending the applicability of the moment equations to non-ideal quadrupole ion traps, the modified versions of the parameterized variables can have additional utility. Calculation of the spatial dependence of ion secular oscillation frequencies is demonstrated as an example. (J Am Soc Mass Spectrom 2006, 17, 889-902) (C) 2006 American Society for Mass Spectrometry
\end{abstract}

$\mathrm{T}$ loday, a large number of analytical mass spectrometers depend exclusively upon electric fields for ion transport, manipulation, and mass analysis. The fields employed may be static (e.g., time-offlight) [1], dynamic (e.g., RF quadrupole [2-5]), or a combination of the two [6-8]. Improvements in performance of RF devices have been realized by changes in the physical configuration and applied potentials [9-12], which produce electric fields of increased complexity (e.g., hexapole, octapole, etc.). Furthermore, ion motion and physicochemical phenomena in such RF devices are influenced by the introduction of a buffer gas at substantial pressure. The high number of ionneutral collisions resulting from extended ion residence times and elevated buffer gas number density can prove beneficial for ion cooling and focusing [13, 14]. In contrast, the average kinetic energy of the ions also can

Published online May 30, 2006

Address reprint requests to Dr. D. E. Goeringer, Oak Ridge National Laboratory, P.O. Box 2008, MS-6131, Oak Ridge, TN 37831, USA. E-mail: goeringerde@ornl.gov be increased significantly above the thermal energy of the neutrals via acceleration in an electric field. The most widely used approach for ion acceleration in electrodynamic ion traps, termed resonance excitation, uses a relatively low-amplitude AC signal at the fundamental frequency of ion axial oscillations applied to the endcaps [15]. An important application of that process in RF multipole devices is collisional activation (CA) [15], in which a portion of the ion-neutral relative (i.e., center-of-mass) kinetic energy is transferred into internal energy of the ions. In such instances, although the kinetic energy associated with any individual collision is generally small, the cumulative effect of multiple collisions enables the collision-induced dissociation (CID) of a wide range of ions [16]. Consequently, many theoretical studies [17-23] and computer simulations [24-29] of confined ion trajectories and the associated ion-neutral collision processes have accompanied analytical applications of RF multipoles.

Rather than using relative kinetic energy and thermal energy in describing gaseous ion transport, it is often practical to consider two directly related quanti- 
ties, the ion effective temperature $[30,31]$ and the buffer gas temperature, respectively. In the context of mass spectrometry, the effective temperature has often been viewed as the temperature characterizing a MaxwellBoltzmann energy distribution of ions that would produce a specified rate for a well-characterized, temperature-dependent ion process $[32,33]$. In the case of the kinetic method [34], the ratio of fragment ion abundances resulting from the dissociation of a protonbound complex, for example, is used to determine the effective temperature. That is, the effective temperature characterizes the behavior of a system with respect to a particular phenomenon rather than the collisional environment itself. In contrast, as indicated in the description below, the effective temperature in this work does describe the state of the system as related to the relative ion-neutral kinetic energy.

Using simple momentum transfer theory, Goeringer and McLuckey [35, 36] have developed phenomenological equations for the effective temperature and CID during resonance excitation in ideal quadrupole ion traps. The effective temperature is a property that also can be determined using ion trajectory simulation techniques to understand ion motion in complicated electric fields $[25,26]$. Such fields typically can be calculated for a variety of device geometries and applied potentials, following which numerical integration of Newton's equations enables simulation of the complicated ion trajectory. The use of various mathematical methods, ranging from constant damping terms to Monte Carlo methods [29, 37-39], allows collisional processes to be approximated in such simulations. The weakness of such trajectory simulation approaches is that investigation of the collective behavior of an ensemble of ions requires trajectory simulations to be performed for multiple ions, either individually or simultaneously.

The two-temperature concept also has been used in applying the Boltzmann equation to calculate for example, collision broadening in ion cyclotron resonance (ICR) [40], ion focusing in field-asymmetric ion mobility spectrometry (FAIMS) [41], and the effective temperature in ideal quadrupole ion traps [42]. The advantage of using the Boltzmann equation is that the relationships between effective temperature and the ion-neutral interaction potential, the ion and neutral masses, and the electric field strength can be established, thereby enabling a priori predictions to be made. However, the resulting system of differential equations is complicated and the collisional terms are difficult to calculate, except in special cases such as the Maxwell model of constant collision frequencies.

Because the Boltzmann equation [43] reflects changes to the ion distribution as a whole, the use of moments of the Boltzmann equation provides a kinetic theory [44] that is intermediary between the momentum-transfer theory and the full use of the Boltzmann equation. The transformation of the Boltzmann equation into moment equations enables the ensemble average value for any property that is a function of the ion velocity to be determined from the corresponding moment. For example, the components of the average ion velocity and energy in the trap, the average ion temperature (effective and kinetic), and the ion number density can each be determined as a function of position and time. Conversely, the moment equations also are subject to limitations of the Boltzmann equation from which they are derived; the significance to this work is that applicable systems are comprised of trace concentrations of atomic ions moving through a pure, dilute buffer gas consisting of unreactive atomic neutrals. It is important to note as well that the moment equations cannot be converted to new differential equations that can be solved to reveal the position of individual ions, as is typically done in ion trajectory simulations. However, such simulations typically require many trajectories to be calculated, either individually or simultaneously, and the resulting data to be collected and further processed to obtain ensemble average values.

Momentum-transfer theory, which is also the simplest approximation to the moment equations, has an inaccuracy of no more than 15-20\% (and in most cases only a few percent) for the transport of atomic ions in atomic gases in the presence of electrostatic fields. Higher-order moment methods, developed from twotemperature [31-45] and multi-temperature [46, 47] approaches to solving the Boltzmann kinetic equation, provide a series of successive approximations for further improving the accuracy. Momentum-transfer and higher-order moment theories have been developed recently for situations where the electric and magnetic fields vary with position as well as with time [48]. Because the typical momentum-transfer collision frequencies are much smaller than the RF frequency of the fields and spatial gradients of the ion number density are much smaller than the spatial variations of the electric field in RF ion traps, it is feasible to apply two-temperature moment theory to the devices. The momentum-transfer approximation, for example, has been deduced from the two-temperature and multitemperature moment theories of ideal quadrupole traps and applied to ion storage in those devices [49]. The purpose of this paper is to present, using sufficient rigor but a minimum of mathematical complexity, the fundamental aspects of the two-temperature moment theory for ion storage and resonance excitation in (ideal and non-ideal) quadrupole ion trap mass spectrometers.

\section{Background and Theory}

\section{Two-Temperature Theory}

In two-temperature theory for drift tubes with electrostatic fields, the total kinetic energy, $K E_{\text {total }}$ of ions accelerated via electric fields through a neutral gas is comprised of terms related to directed and stochastic motion. The directed energy is obtained from the electric field and exhibited as ion drift motion along the field. The stochastic energy is acquired from the thermal 
energy of the neutrals and from the electric field, but transferred into random motion via ion-neutral collisions, and is equal to the relative (or center-of-mass) kinetic energy, $K E_{r e l}$. It is now well-established that $K E_{\text {total }}$ is given by the expression in eq 1 [44], where \langle\rangle denotes an ensemble average.

$$
\begin{aligned}
\left\langle K E_{\text {total }}\right\rangle & =\left\langle\frac{1}{2} m \mathrm{v}^{2}\right\rangle \\
& =\left\langle\frac{1}{2} M \mathrm{~V}^{2}\right\rangle+\frac{1}{2} m \mathrm{v}_{\mathrm{d}}^{2}+\frac{1}{2} M \mathrm{v}_{\mathrm{d}}^{2}(1+\beta)
\end{aligned}
$$

In the above equation, $m, \mathbf{v}_{\mathrm{d}}$, and $\mathrm{v}$ are the ion mass, drift speed, and instantaneous speed, respectively, and the neutral mass and instantaneous speed are $m$ and $V$, respectively. The correction term, $\beta$, is zero for momentum-transfer theory and the Maxwell ion-neutral interaction model (see below), and in general has a magnitude about 0.1 for other types of interactions [50]. The first term on the right hand side of eq 1 is simply the thermal energy of the neutrals, which is equal to $(3 / 2) k_{B} T$, where $T$ is the neutral temperature and $k_{B}$ is the Boltzmann constant. For situations in which $m \gg$ $M$, most of the electric field energy is manifested in the second term in eq 1 as increased $\mathrm{v}_{\mathrm{d}}$, whereas the last term in eq 1 can contribute to additional ion heating for other cases.

It is straightforward to transform eq 1 into the center-of-mass for an ion-neutral collision to give eq 2 .

$$
\left\langle K E_{\text {rel }}\right\rangle=\frac{3}{2} k_{B} T+\frac{3}{2} M v_{d}^{2}\left(1+\frac{M}{m+M} \beta\right)=\frac{3}{2} k_{B} T_{\text {eff }}
$$

Two-temperature theory thus indicates that the separate effects of thermal heating and electric field acceleration of ions can be combined into a single parameter, the effective temperature, $T_{\text {eff, }}$, that is directly related to $\left\langle K E_{\text {rel }}\right\rangle$. Furthermore, ions present in trace amounts can have $T_{\text {eff }} \gg T$, because electric fields can increase $\left\langle K E_{\text {rel }}\right\rangle$ to values considerably greater than thermal energy. By using the above equations, and the relationship, $K E_{\text {total }}$ $=(3 / 2) k_{B} T_{i o n}$, the expression for $T_{\text {eff }}$ also can be rewritten in terms of the ion temperature, $T_{i o n}$, as indicated in eq 3.

$$
T_{\text {eff }}=\frac{m T+M T_{\text {ion }}}{m+M}
$$

The drift velocity is constant for situations in which the momentum-transfer collision frequency for ions and neutrals is much greater than the rate of change in the electric field, and for which the electric field strength is not a function of position. Neither condition is satisfied for the typical operating conditions encountered in ion trap mass spectrometry. For example, at typical helium buffer gas pressures of 0.1-1.0 mTorr, the momentum- transfer collision frequency is about $10^{2}-10^{3} \mathrm{~Hz}$, compared with normal RF frequencies $(\Omega / 2 \pi)$ of $\sim 0.7-2$ $\mathrm{MHz}$. Except for ion-neutral collisions that can be described by the Maxwell model, the momentumtransfer collision frequency also is not constant (see below) but instead is a complicated function of $m, M$, $T_{\text {eff, }}$ the interaction potential, position, and time. Furthermore, the fast RF ion motion modulates ion secular oscillations that occur at lower frequencies, which are the result of spatial variations in the electric field. Besides the energy-transfer that occurs between colliding ion-neutral pairs, collisions also can change the phase of these oscillations resulting in RF energy gain or loss and a concomitant change in oscillation amplitude. The result is that the simple phenomenological eqs 1-3 are inadequate for treating ion motion accurately in such devices.

We have developed a more detailed kinetic theory for ion traps, based on moments of the Boltzmann equation, which is summarized below. Parameterized forms of the moment equations also are formulated using the electric fields associated with ion trapping and dipolar excitation in both ideal and commonly used ion trap configurations. In addition, a more thorough evaluation of the momentum-transfer collision frequency is made by treating such collisions with the same detail as used for electric fields. Expressions are developed for specific ion-neutral interactions governed by Maxwell and rigid-sphere models and by more general potential functions that vary with the separation distance.

\section{Moment Treatment of the Boltzmann Equation}

The Boltzmann equation [43-51] is a fundamental kinetic equation describing the effect of external fields and ion-neutral collision processes on the time-dependent behavior of an ion distribution function, $f$, in the 6-dimensional phase space of ion velocity, $\mathbf{v}$, and position, $\mathrm{r}$. Thus, $f(\mathbf{r}, \mathbf{v}, t) \mathrm{d} \mathbf{r d} \mathbf{v}$ is the number of ions at time $t$, in the volume element $d \mathbf{r}$ near $\mathbf{r}$, having velocities in the interval $\mathbf{d} \mathbf{v}$ near $\mathbf{v}$. In ion traps, the electric field, $\mathbf{E}$, is position- and time-dependent, and is therefore written as a function of $\mathbf{r}$ and $t$. Note also that average velocity and acceleration are not equal necessarily to the timederivatives of average ion position and velocity, respectively, because $\mathbf{r}$ and $\mathbf{v}$ are independent vectors in phase space. Rather, the coupling of ion position, velocity, and acceleration arises via changes in $f(\mathbf{r}, \mathbf{v}, t)$ due to ion motion, external electric fields, and ion-neutral collisions. The ions are assumed to be present only in trace amounts in a pure atomic gas, so that only the electric fields and ion-neutral collisions affect the ion distribution function. Furthermore, the neutral gas distribution function, which is independent of time or position in the apparatus, has an equilibrium Maxwellian form characterized by the gas temperature, $T$.

Because the Boltzmann equation reflects changes to the ion distribution as a whole, it is not possible to 
determine the trajectory, or any other property, of a single ion. Instead, for any function of ion velocity, $\varphi(\mathbf{v})$, the average value of that property for the ion ensemble can be determined from the moment, $\bar{\varphi}_{i}$, with respect to $f$, as in eq 4 .

$$
\overline{\varphi_{i}(\mathbf{r}, t)}=\frac{\int \varphi_{i}(\mathbf{v}) f(\mathbf{r}, \mathbf{v}, t) \mathrm{d} \mathbf{v}}{\int f(\mathbf{r}, \mathbf{v}, t) \mathrm{d} \mathbf{v}}
$$

These moments include, for example, the average ion velocity given by eq 5 and the average kinetic energy given by eq 6 .

$$
\begin{aligned}
\langle\mathbf{v}(\mathbf{r}, t)\rangle=\overline{\mathbf{v}(\mathbf{r}, t)} & =\frac{\int \mathbf{v} f(\mathbf{r}, \mathbf{v}, t) \mathrm{d} \mathbf{v}}{n(\mathbf{r}, t)} \quad \varphi(\mathbf{v})=\mathbf{v} \\
\left\langle K E_{\text {ion }}(\mathbf{r}, t)\right\rangle= & \frac{1}{2} m[v(\mathbf{r}, t)]^{2} \\
= & \frac{\int\left\lfloor\frac{1}{2} m \mathbf{v} \cdot \mathbf{v}\right\rfloor f(\mathbf{r}, \mathbf{v}, t) \mathrm{d} \mathbf{v}}{n(\mathbf{r}, t)} \\
\varphi(\mathbf{v}) & =\frac{1}{2} m \mathbf{v} \cdot \mathbf{v}
\end{aligned}
$$

Here, the number density of ions, $n$, is given by eq 7 .

$$
n(\mathbf{r}, t)=\int f(\mathbf{r}, \mathbf{v}, t) \mathrm{d} \mathbf{v}
$$

Thus, knowledge of the distribution function would enable any moment (or related quantity) to be found via integration. However, solving the Boltzmann equation directly for $f$ is generally not possible. An alternative method for determining moments involves transforming the Boltzmann equation into a differential equation that describes the desired moments themselves [44], thus making it possible to find the corresponding properties without determining $f$ directly.

The transformation, which involves multiplying the Boltzmann equation from the left by $\varphi(\mathbf{v})$ and integrating over $\mathbf{v}$, produces a partial differential equation for $\bar{\varphi}_{i}(\mathbf{r}, t)$. Using $\varphi=1$ yields the equation of continuity that expresses the fact that ions are neither created nor destroyed in the apparatus, since neither the possibility of ion sources or sinks nor of ion-neutral reactions are considered in this work. The equation of continuity then enables the time derivative of $n(\mathbf{r}, t)$ to be eliminated from the transformed Boltzmann equation. Finally, terms involving the difference between the product of two averages and the average of their product, $\left(\bar{\varphi}_{i} \overline{\mathbf{v}}-\overline{\varphi_{i} \mathbf{v}}\right)$, and the difference between two similar quantities, $\left(\bar{\varphi}_{i} \nabla \cdot \overline{\mathbf{v}}-\nabla \cdot \overline{\mathbf{v} \varphi_{\mathrm{i}}}\right)$, which both appear in the transformed equation, will likely be small. The resulting approximation for the general moment equation is given by eq 8 , which is an ordinary differential equation.

$$
\frac{\mathrm{d}}{\mathrm{d} t} \bar{\varphi}_{i}-\frac{e}{m} \overline{\mathbf{E} \cdot \nabla_{\mathrm{v}} \varphi_{i}}=\overline{\hat{\jmath} \varphi_{i}}
$$

Here, $e$ is the ion charge, and the explicit indication of time and position dependence has been dropped. The action of the adjoint, $\hat{\jmath}$, of the Boltzmann collision operator can be thought of as producing the average rate of change in $\varphi$ due to collisions with neutrals. Eq 8 is actually an entire system of ordinary differential equations for the set of functions $\varphi_{i}$ in $\mathbf{v}$-space, since for each $\varphi_{i}$, evaluation of $\overline{\hat{J} \varphi_{i}}$ usually involves expansion as a linear combination of other members of the set. The equation for each moment $\bar{\varphi}_{i}$ can then be solved, in principle, only if the higher order moments (i.e., $\left.\varphi_{i+1}, \varphi_{i+2}, \ldots\right)$ are known. Because the system of expressions represented by the general moment equation is infinite, it has to be closed by some type of truncation procedure based on properties of $\hat{J} \varphi_{i}$ [48].

\section{Moment Equation for Effective Temperature}

As previously discussed, the primary quantity of interest in this work is $T_{\text {eff. }}$. The desired velocity moment is $\varphi(\mathbf{v})=\frac{1}{2} m \mathbf{v} \cdot \mathbf{v}$, since $T_{\text {eff }}$ is related to $\frac{1}{2} m \mathrm{v}^{2}$ via eqs 1,2 , and 3 . The corresponding moment equation (for total kinetic energy) obtained from eq 8 is eq 9 .

$$
\frac{d}{d t} \overline{\frac{1}{2} m \mathbf{v}^{2}}-\frac{e}{m} \mathbf{E} \cdot \overline{m \mathbf{v}}=\overline{\hat{J}-m v^{2}}
$$

Evaluation of $\overline{\hat{J} \varphi_{i}}$ via the matrix representation of the collision operator is generally used [48] when results of the highest accuracy are desired. An alternative approach that gives the same result is to use an approximation method based on momentum transfer theory, in which the momentum and energy gained from the electric field by the ion is balanced at steady-state by losses (of momentum and energy) due to ion-neutral collisions.

In this approach, the right hand side of eq 9 is equated to the average rate of energy-transfer. From the condition for energy balance, that rate can be approximated by multiplying the average energy transferred from an ion to a neutral upon their collision, $\frac{1}{2} \mu\left\langle g^{2}\right\rangle$. $(1-\cos \theta)$, (where $\mu$ is the ion-neutral reduced mass, and $g$ is their relative speed) by the average collision rate, $\langle\nu\rangle$. Summing all collisions by integrating over all collision angles, $\theta$, then gives eq 10 [44].

$$
\overline{\hat{J} \frac{1}{2} m \mathrm{v}^{2}}=-2 \frac{m M}{(m+M)^{2}} \overline{\left(\frac{1}{2} m \mathrm{v}^{2}-\frac{1}{2} M \mathrm{~V}^{2}\right)} \nu
$$

Substituting eq 10 into eq 9 yields eq 11, where the momentum-transfer collision frequency, $\xi$, is given by eq 12. 


$$
\begin{aligned}
& \frac{\partial}{\partial t} \overline{\frac{1}{2} m \mathrm{v}^{2}}-e \mathbf{E} \cdot \overline{\mathbf{v}}+\frac{2 m \xi}{m+M} \overline{\left(\frac{1}{2} m \mathrm{v}^{2}-\frac{1}{2} M \mathrm{~V}^{2}\right)}=0 \\
& \xi=\frac{M}{m+M} \nu
\end{aligned}
$$

Finally, eq 11 (and eq 9 as well) can be converted to the desired (ordinary) differential equation for the moment of ion effective temperature by using eqs 1,2 , and 3 . The result is given by eq 13 , where $\varepsilon=T_{\text {eff }} / T$.

$$
\frac{\mathrm{d}}{\mathrm{d} t} \varepsilon-\frac{2 \mu}{3 k_{B} T} \frac{e}{m} \mathbf{E} \cdot \overline{\mathbf{v}}+\frac{2 m \xi}{m+M}[\varepsilon-1]=0
$$

Thus, solution of the moment equation for effective temperature (or energy) requires another moment equation, one describing variations in $\overline{\mathbf{v}}$ or a related quantity.

\section{Moment Equation for Ion Velocity}

Proceeding in a similar manner to that used to derive the moment equation for energy via momentum transfer theory, momentum balance dictates that the average rate of momentum transfer is given by $\hat{J} m \mathbf{v}$. This average rate, in turn, can be approximated by multiplying the average momentum transferred from an ion to a neutral upon their collision, $\mu\langle g\rangle(1-\cos \theta)$ by the average collision rate. Further, realizing that averaging over all collisions gives $\langle g\rangle=\overline{\mathbf{v}}$ and then integrating over all collision angles leads to eq 14 [44].

$$
\overline{\widehat{J} m \mathbf{v}}=-\mu \nu \overline{\mathbf{v}}
$$

The required moment equation is obtained by using $\varphi$ $=m \mathbf{v}$ in eq 8 to give eq 15 .

$$
\frac{\partial}{\partial t} \overline{m \mathbf{v}}-e \mathbf{E}=\overline{\hat{J} m \mathbf{v}}
$$

Substitution of eq 14 into eq 15 and dividing through by $m$ leads to eq 16 .

$$
\frac{\mathrm{d}}{\mathrm{d} t} \overline{\mathbf{v}}-\frac{e}{m} \mathbf{E}+\xi \overline{\mathbf{v}}=0
$$

Because eq 16 is a vector equation, it can be rewritten as three ordinary differential equations for the moment of ion velocity in each of the Cartesian coordinate directions, $\overline{\mathrm{v}}_{x}, \overline{\mathrm{v}_{y}}$, and $\overline{\mathrm{v}}_{z}$ as presented in eq 17 .

$$
\frac{\mathrm{d}}{\mathrm{d} t} \overline{\mathrm{v}_{u}}-\frac{e}{m} E_{u}+\overline{\xi \mathrm{v}_{u}}=0, \quad u=x, y, z
$$

Thus, eqs 13 and 17 constitute a complete system of four differential equations that can be solved directly (normally, by numerical methods) for the average velocity along three mutually perpendicular directions in the apparatus, for the effective temperature (or equivalently, center-of-mass energy), and for the ion number density, each as a function of time and position.

\section{Physical Configurations and the Associated Electric Fields}

In this paper, all quadrupole ion traps are assumed to be cylindrically symmetric about the $z$-axis, and to have the potential $\phi_{R}$ in eq 18 applied to the ring electrode (internal radius $r_{0}$ ).

$$
\phi_{R}=U_{R}+V_{R} \cos (\Omega t)
$$

Here the amplitude of the DC component is $U_{R}$; the zero-to-peak amplitude of the AC component is $V_{R}$ and its angular frequency is $\Omega$. The identical endcaps, spaced a distance $2 z_{0}$ apart, are arranged symmetrically with respect to the trap center. These variables are generally combined to give the dimensionless parameters $a$ and $q$, which are defined in eqs 19a and b.

$$
\begin{aligned}
& a_{z}=-2 a_{u}=\frac{-16 e U_{R}}{m\left(r_{0}^{2}+2 z_{0}^{2}\right) \Omega^{2}} \quad u=x, y \\
& q_{z}=-2 q_{u}=\frac{8 e V_{R}}{m\left(r_{0}^{2}+2 z_{0}^{2}\right) \Omega^{2}} \quad u=x, y
\end{aligned}
$$

In the resonance excitation technique, the supplementary potential $\phi_{D}$ in eq 20 normally is applied $180^{\circ}$ out-of-phase (i.e., in a dipolar fashion) to each endcap.

$$
\phi_{D}=\left[U_{D}+V_{D} \cos (\omega t+\delta)\right] / 2
$$

Here $U_{D}$ is the amplitude of its dc component and $V_{D}$ is the peak-to-peak amplitude, $\omega$ the angular frequency, and $\delta$ the phase shift of its ac component. In this work, $U_{D}$ and $V_{D}$ also are combined to give the new dimensionless parameters $b$ and $d$, defined in eqs 21a and b).

$$
\begin{aligned}
& b_{z}=-2 b_{u}=\frac{-16 e U_{D}}{m\left(r_{0}^{2}+2 z_{0}^{2}\right) \Omega^{2}} \quad u=x, y \\
& d_{z}=-2 d_{u}=\frac{8 e V_{D}}{m\left(r_{0}^{2}+2 z_{0}^{2}\right) \Omega^{2}} \quad u=x, y
\end{aligned}
$$

The total electric potential, $\Phi(x, y, z, t)$ in the quadrupole ion trap can be expressed by a multipole expansion of the form shown in eq 22 [52].

$$
\begin{aligned}
\Phi(x, y, z, t)= & \phi_{R} \sum_{l=0}^{\infty} A_{2 l}^{R}\left(\frac{r}{r_{0}}\right)^{2 l} P_{2 l}\left(\frac{z}{r}\right) \\
& +\phi_{D} \sum_{l=0}^{\infty} A_{2 l+1}^{D}\left(\frac{r}{r_{0}}\right)^{2 l+1} P_{2 l+1}\left(\frac{z}{r}\right)
\end{aligned}
$$


Table 1. Properties for selected ion trap configurations

\begin{tabular}{cccc}
\hline & \multicolumn{3}{c}{ Ion trap configuration } \\
\cline { 2 - 4 } Parameter & Ideal & ITMS & LCO \\
\hline \hline$r_{0}(\mathrm{~cm})$ & 1.000 & 1.000 & 0.707 \\
$z_{0}(\mathrm{~cm})$ & 0.707 & 0.783 & 0.783 \\
$\Omega / 2 \pi(\mathrm{MHz})$ & 1.0 & 1.1 & 0.76 \\
$A_{1}{ }^{D}$ & 0.568 & 0.4929 & 0.2151 \\
$A_{2}{ }^{R}$ & -1 & -0.8945 & -0.5744 \\
$A_{3}{ }^{D}$ & 0.278 & 0.2394 & 0.0978 \\
$A_{4}{ }^{R}$ & 0 & -0.0158 & -0.0044 \\
$A_{5}{ }^{D}$ & -0.018 & -0.0234 & -0.0225 \\
$A_{6}{ }^{R}$ & 0 & -0.0076 & -0.0021 \\
\hline
\end{tabular}

The dimensionless expansion coefficients, $A_{l}$, for the Legendre polynomials, $P_{l}$, of order, $l$, are dependent on the specific physical configuration of the ion trap. The $A_{l}$ values, obtained from the ion trap simulation program ITSIM [25, 26], are shown in Table 1 for an ideal trap, a Finnigan ITMS "stretched" trap, and a Finnigan LCQ trap. The $R$ and $D$ superscripts associated with $A_{l}$ indicate that the terms involve the ring and endcap electrodes, respectively. In addition, the standard values for $r_{0}, z_{0}$, and $\Omega$ are indicated.

The three components of the electric field are obtained by partial differentiation of $\Phi(x, y, z, t)$. The results are shown in eqs $23 a$ and $b$ ).

$$
\begin{aligned}
-\frac{e}{m} E_{u}= & \frac{\Omega^{2}}{4}\left(\left[\widetilde{a}_{u}-2 \widetilde{q}_{u} \cos (\Omega t)\right] u\right. \\
& \left.+\left[\widetilde{b}_{u}-2 \widetilde{d}_{u} \cos (\omega t+\delta)\right] \frac{u z}{r_{0}}\right), u=x, y \\
-\frac{e}{m} E_{z}= & \frac{\Omega^{2}}{4}\left(\left[\widetilde{a}_{z}-2 \widetilde{q}_{z} \cos (\Omega t)\right] z\right. \\
& \left.+\left[\widetilde{b}_{z}-2 \widetilde{d}_{z} \cos (\omega t+\delta)\right] r_{0}\right)
\end{aligned}
$$

In eqs $23 \mathrm{a}$ and $\mathrm{b}$ the dimensionless parameters $\tilde{a}, \tilde{q}, \tilde{b}$, and $\widetilde{d}$ (defined in Appendix A) are similar to $a, q, b$, and $d$, but they are modified by terms that include distance from the trap center and the expansion coefficients to take into account nonlinear variations with position and differences in configuration. Note that $\widetilde{a}$ and $\widetilde{q}$ become equal to $a$ and $q$, respectively, for an ideal trap, since $A_{4}^{R}, A_{6}^{R}=0, A_{2}^{R}=-1$ and $z_{0}=r_{0} / \sqrt{2}$ in that case. However, because the endcaps are hyperbolic, the dipole term, $A_{1}^{D} \neq 1$ and the terms, $A_{3}^{D}, A_{5}^{D} \neq 0$, as would be the case for an ideal dipole field. It is also interesting to note that even at the trap center, $\tilde{a} \neq a$ and $\tilde{q} \neq q$ for the nonlinear traps because $A_{2}^{R} \neq-1$ and $z_{0} \neq$ $r_{0} / \sqrt{2}$

Substituting the expressions for $E_{u}$ given in eqs 23a and $b$ ) into the moment eqs 13 and 17 , produces eqs 24 , $25 a$ and $b$ ).

$$
\begin{aligned}
\frac{\mathrm{d}}{\mathrm{d} t} \varepsilon & +\frac{\mu \Omega^{2}}{6 k_{B} T} \sum_{u=x, y, z}\left[\tilde{a}_{u}-2 \widetilde{q}_{u} \cos (\Omega t)\right] u \overline{\mathrm{v}_{u}} \\
& +\frac{2 m \xi}{m+M}(\varepsilon-1) \\
& +\frac{\mu \Omega^{2}}{6 k_{B} T}\left\{\left[\tilde{b}_{x}-2 \tilde{d}_{x} \cos (\omega t+\delta)\right] \frac{x z}{r_{0}} \overline{\mathrm{v}}_{x}\right. \\
& +\left[\widetilde{b}_{y}-2 \widetilde{d}_{y} \cos (\omega t+\delta)\right] \frac{y z}{r_{0}} \overline{\mathrm{v}}_{y} \\
& \left.+\left[\tilde{b}_{z}-2 \tilde{d}_{z} \cos (\omega t+\delta)\right] r_{0} \overline{\mathrm{v}}_{z}\right\}=0
\end{aligned}
$$

$$
\begin{aligned}
\frac{\mathrm{d}}{\mathrm{d} t} \overline{\mathrm{v}_{u}}+ & \frac{\Omega^{2}}{4}\left(\left[\widetilde{a}_{u}-2 \widetilde{q}_{u} \cos (\Omega t)\right] u\right. \\
+ & {\left.\left[\widetilde{b}_{u}-2 \widetilde{d}_{u} \cos (\omega t+\delta)\right] \frac{u z}{r_{0}}\right)+\overline{\xi \mathrm{v}_{u}}=0, } \\
u & =x, y \\
\frac{\mathrm{d}}{\mathrm{d} t} \overline{\mathrm{v}_{z}}+ & \frac{\Omega^{2}}{4}\left(\left[\widetilde{a}_{z}-2 \widetilde{q}_{z} \cos (\Omega t)\right] z\right. \\
+ & {\left.\left[\widetilde{b}_{z}-2 \widetilde{d}_{z} \cos (\omega t+\delta)\right] r_{0}\right)+\overline{\xi \mathrm{v}_{z}}=0 }
\end{aligned}
$$

When $\xi$ is constant (see the Maxwell model, below), eqs $25 \mathrm{a}$ and b) can be solved analytically. Consequently, inserting the solutions for all $\mathrm{v}_{u}$ values into eq 24 gives an ordinary differential equation that also can be solved analytically for $\varepsilon$. Both analytical solutions are given in Appendix B.

\section{Ion-Neutral Collisions}

From momentum-transfer theory, $\xi$ is given by eq 26 , where $N$ is the neutral number density and $\langle\nu\rangle$ is equal to $N\langle g\rangle Q^{(1)}$.

$$
\xi\left(\left\langle K E_{r e l}\right\rangle\right)=\frac{M}{m+M} N\langle g\rangle Q^{(1)}\left(\left\langle K E_{r e l}\right\rangle\right)
$$

The momentum-transfer cross section, $Q^{(1)}$, taken at average energy $\left\langle K E_{\text {rel }}\right\rangle$, is defined as an integral over the impact parameter, $b$ [53]. Momentum-transfer theory assumes a Langevin or Maxwell collision model for which the interaction potential has an inverse-fourth power (attractive) potential. In that case, the classicalmechanical expression for $Q^{(1)}$, given in eq 27, is essentially exact.

$$
Q^{(1)}=2.210 \pi\left(\frac{\alpha e^{2}}{2 K E_{r e l}}\right)^{\frac{1}{2}}
$$

In the above equation, $\alpha$, is the polarizability of the neutral. It should be noted that the value calculated for 
$Q^{(1)}$ from eq 27 is approximately $10 \%$ larger than that calculated for orbiting collisions, because the former takes into account trajectories for which $b$ is greater than the critical impact parameter. Since $Q^{(1)}$ is inversely proportional to $g$ in this case, the result is that $\xi$ is constant for momentum-transfer theory.

Within the context of two-temperature theory, the momentum-transfer collision frequency is defined by eq 28 , which is the analog of eq 26 .

$$
\xi\left(T_{\text {eff }}\right)=\frac{4}{3} \frac{M}{m+M} N\left(\frac{8 k_{B} T_{\text {eff }}}{\pi \mu}\right)^{1 / 2} \bar{\Omega}^{(1,1)}\left(T_{\text {eff }}\right)
$$

In this instance $Q^{(1)}\left(\left\langle K E_{\text {rel }}\right\rangle\right)$ is replaced by the collision integral, $\bar{\Omega}^{(1,1)}\left(T_{\text {eff }}\right)$, obtained by averaging $Q^{(1)}\left(K E_{\text {rel }}\right)$ over the distribution of $K E_{r e l}$ values. For the Maxwell model, the quantity $\left(T_{\text {eff }}\right)^{1 / 2} \bar{\Omega}^{(1,1)}$ is independent of $T_{\text {eff. }}$ Therefore, as with the momentum-transfer theory, $\xi$ in the twotemperature theory is constant for Maxwell interactions.

For classical-mechanical rigid-sphere collisions, $Q^{(1)}$ and $\overline{\Omega^{(1,1)}}\left(T_{\text {eff }}\right)$ are constant (and equal to $\pi d^{2}$ for rigid spheres of diameter $d$ ) instead of $\xi$. Consequently, eq 28 can be rewritten as eq 29 , indicating that $\xi$ is dependent on $T_{\text {eff }}$ (or equivalently $K E_{\text {rel }}$ ) for that collision model.

$$
\begin{aligned}
\xi\left(T_{\text {eff }}\right) & =\frac{4}{3} \frac{M}{m+M} N\left(\frac{8 k_{B} T}{\pi \mu}\right)^{1 / 2} \bar{\Omega}^{(1,1)}\left(T_{\text {eff }}\right)\left(\frac{T_{\text {eff }}}{T}\right)^{1 / 2} \\
& =\xi_{0}\left(\frac{T_{\text {eff }}}{T}\right)^{1 / 2}
\end{aligned}
$$

In the case of general ion-neutral interactions, for which the interaction potential has been determined via $a b$ initio methods, it is now routine to calculate values for $Q^{(1)}\left(K E_{r e l}\right)$ by numerical techniques $[54,55]$. The corresponding collision integral can then be obtained by averaging as before; subsequent substitution of $\bar{\Omega}^{(1,1)}\left(T_{\text {eff }}\right)$ into eq 29 gives $\xi\left(T_{\text {eff }}\right)$.

Because $\xi$ is constant for ion-neutral interactions governed by the Maxwell model, the velocities $\overline{\mathrm{v}}_{u}$ in equations (24) and $(25 a, b)$ are independent of each other in this case. However, for rigid-sphere collisions and the type of general ion-neutral interactions noted above, $\xi$ is dependent on $T_{\text {eff }}$ (or equivalently $K E_{\text {rel }}$ ). Thus, the moment equations eqs 13 and 17 are coupled by $\xi$ in this situation, thereby allowing kinetic energy to be exchanged among the three components of velocity via collisions.

\section{Results and Discussion}

The following examples, although not comprehensive, will illustrate some of the capabilities of moment theory for predicting the characteristics of ion ensembles during various processes in ion traps. Specific characteristics calculated from eqs 13 and 17 include the positionand time-dependent effective temperature, $T_{\text {eff, }}$ associ- ated with ion storage and resonance excitation [recall that the explicit indication of time and position dependence for $\varphi(\mathbf{r}, t)$ (e.g., $T_{\text {eff }}(\mathbf{r}, t)=\varepsilon(\mathbf{r}, t) \cdot T$ was dropped in eq 8 , but in fact $T_{\text {eff }}$ does represent an ensemble average over the ion population at a specific $r$ and instantaneous t]. It is important to note that in these and other calculations described below, the rather narrow range of distances used $(0 \leq z \leq 1 \mathrm{~mm}$ from the trap center) does not imply that ion trajectories are limited only to those regions of the trap. Rather, the implication is that the relatively small effects on effective temperature seen in regions of low electric field strength can be expected to be more pronounced as ions move farther from the trap center. The calculations were performed initially in Mathcad 12 by coding the analytical solution for $T_{\text {eff }}$ given in eq B.14, and the results were then verified using the Mathcad differential equation functions to solve eqs 13 and 17. In all examples, the buffer gas (He) temperature and pressure were $T=300 \mathrm{~K}$ and 0.001 torr, respectively, and the ions were $m / z=100$. The Maxwell model was used for ion-neutral collisions. Values of other pertinent parameters are indicated as required below.

\section{Fundamental Frequencies of Ion Oscillation}

In an ideal trap the fundamental circular, $f_{u, 0}^{\text {ideal }}$, and angular, $\omega_{u, 0}^{\text {ideal }}$, frequencies of oscillation, given by eq 30 , are position-independent.

$$
f_{u, 0}^{\text {ideal }}=\frac{\omega_{u, 0}^{\text {ideal }}}{2 \pi}=\frac{\beta_{u}}{2} \frac{\Omega}{2 \pi} \quad u=x, y, z
$$

The coefficient $\beta_{u}$ is given by a continued fraction expression [5] in terms of $a_{u}$ and $q_{u}$. However, for a non-ideal trap, $f_{u, 0}$ varies with the amplitude of ion oscillation due to the nonlinear electric fields. This variation can be calculated using eq 31 in which the fundamental frequencies are written in a modified form, $\tilde{f}_{u, 0}$, that is a function of $\widetilde{a}_{u}$ and $\widetilde{q}_{u}$, and thus implicitly also a function of the ion location (see Appendix A). Note that $\widetilde{\beta}_{u}$ is calculated from the same continued fractions expression as $\beta_{u}$, but with $\widetilde{a}_{u}$ and $\widetilde{q}_{u}$ substituted for $a_{u}$ and $q_{u}$.

$$
\tilde{f}_{u, 0}(x, y, z)=\frac{\widetilde{\omega}_{u, 0}(x, y, z)}{2 \pi}=\frac{\widetilde{\beta}_{u}\left(\widetilde{a}_{u}, \widetilde{q}_{u}\right)}{2} \frac{\Omega}{2 \pi} \quad u=x, y, z
$$

The variation of $\tilde{f}_{u, 0}$ with specific ion trap configuration also can be determined, since $\widetilde{a}_{u}$ and $\widetilde{q}_{u}$ are functions of the expansion coefficients, $A_{l}$, which differ for the shape and spacing of electrodes (see Table 1 ). Figure 1 shows values of $\tilde{f}_{z, 0}$ (normalized by $\Omega / 2 \pi$ ) at $q_{z}=0.3$, calculated as a function of axial distance from the trap center for the ITMS, ideal, and LCQ configurations. The equations used for the calculations (eqs 31 and A.1a and b), 


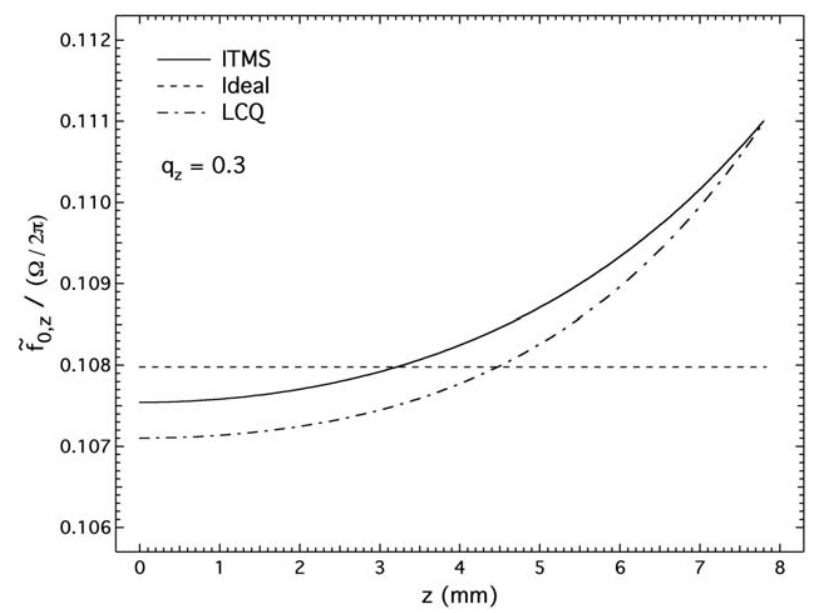

Figure 1. Variation in the fundamental axial (circular) frequency, $f_{z, 0}$, for trapped ions at $q_{z}=0.3$, normalized to the trapping RF (circular) frequency, $\Omega / 2 \pi$, calculated as a function of axial position, $z, z=0$ at the trap center) for the ITMS, ideal, and LCQ configurations.

along with the continued fraction expression for $\widetilde{\beta}_{u}$ were coded in Mathcad 12 . Because $\widetilde{\beta}_{z}$ does not vary with axial position in the ideal trap, $\tilde{f}_{z, 0}$ is invariant over the indicated $z$ range. Over the same axial distance, the relative variation in the normalized value of $\tilde{f}_{z, 0}$ is slightly larger for the LCQ than for the ITMS.

\section{Ion Storage}

In the collision-dominated regime of RF ion traps, ion power absorption from the oscillating electric fields can lead to an increase in their effective temperature [35, 36]; the process is known as RF heating when the power source is the main RF trapping field. Thus, during ion trapping the increase in $T_{\text {eff }}$ due to RF heating should be a function of: (1) $q_{u=x, y, z}$ (or equivalently, $V_{R}$ ) at a fixed distance from the trap center, and (2) distance from the trap center at a fixed $q_{u=x, y, z}$, because the electric field in ion traps varies with each (see eq 23). Figure 2 shows the variation in $\hat{T}_{\text {eff }}$ at an axial distance $z=0.25 \mathrm{~mm}$ from the trap center, calculated as a function of $q_{z}$ for the ideal, ITMS, and LCQ traps in which only the ring $\mathrm{RF}$ voltage is applied. The plotted $\hat{T}_{\text {eff }}$ points represent the time average of $T_{\text {eff }}$ over 10 cycles of $\tilde{f}_{z, 0}$ corresponding to the relevant $q_{z}$. Note that although ion trapping does not occur at $q_{z}=0$ since the electric field is zero, $\hat{T}_{\text {eff }}$ is equal to $T$ in this case due to thermal heating from ion-neutral collisions. The plot in Figure 3 compares $\hat{T}_{\text {eff }}$ values at $q_{z}=0.3$, calculated as a function of axial distance $(z=0-1 \mathrm{~mm})$ from the trap center, for the same trap configurations and applied voltage as in Figure 2. Each plotted $\hat{T}_{\text {eff }}$ data point again represents the time average over ten cycles of $\tilde{f}_{z, 0}$ at $q_{z}=0.3$. A delay time of $3 / \xi$, which is about $4 \mathrm{~ms}$ for ions of $\mathrm{m} / z=$

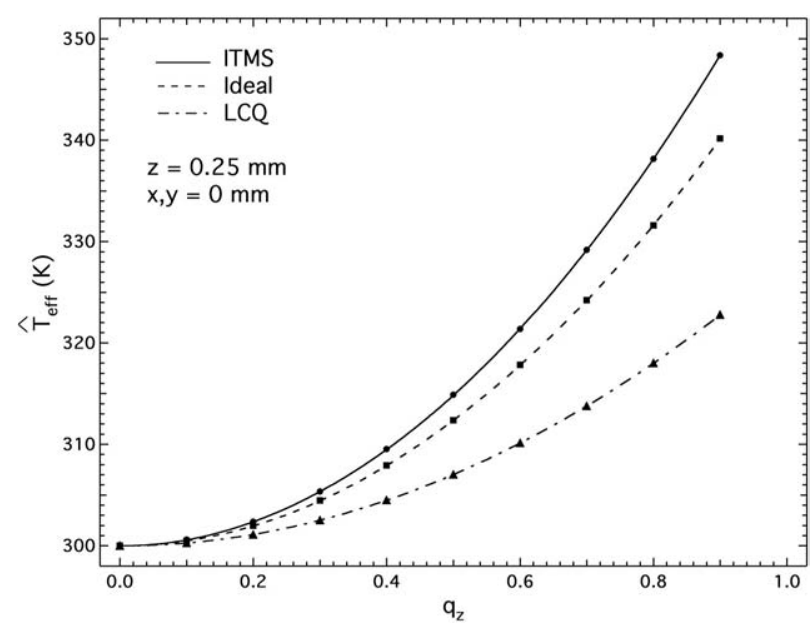

Figure 2. The time-averaged ion effective temperature, $\hat{T}_{\text {eff }}$ at an axial distance of $z=0.25 \mathrm{~mm}$ from the trap center, calculated for the Maxwell model of ion-neutral interactions as a function of $q_{z}$. The individual curves represent results for three different ion trap configurations in which only the ring RF voltage is applied: ideal, ITMS, and LCQ. Each plotted point is the time average over 10 cycles of the fundamental axial frequency at the relevant $q_{z}$ (see text), after a delay time of $3 / \xi$ (about $4 \mathrm{~ms}$ for $\mathrm{m} / \mathrm{z} 100$ ions in $\mathrm{He}$ at $1 \mathrm{mTorr}$ ).

100 and a He pressure of 1 mTorr, preceded each averaging step in both Figures 2 and 3.

For each $q_{z}$ curve in Figure $3, \hat{T}_{\text {eff }}$ is $300 \mathrm{~K}$ at the trap center because $E_{z}$ is zero at that location (there is no contribution from $E_{u=x, y}$ along the $z$-axis); ion heating again results only from thermal motion of the neutrals. As expected, $\hat{T}_{\text {eff }}$ increases with $q_{z}$ (Figure 2 ) and with axial distance (Figure 3) from the trap center due to the increase in $E_{z}$ with those parameters. The $\hat{T}_{\text {eff }}$ results

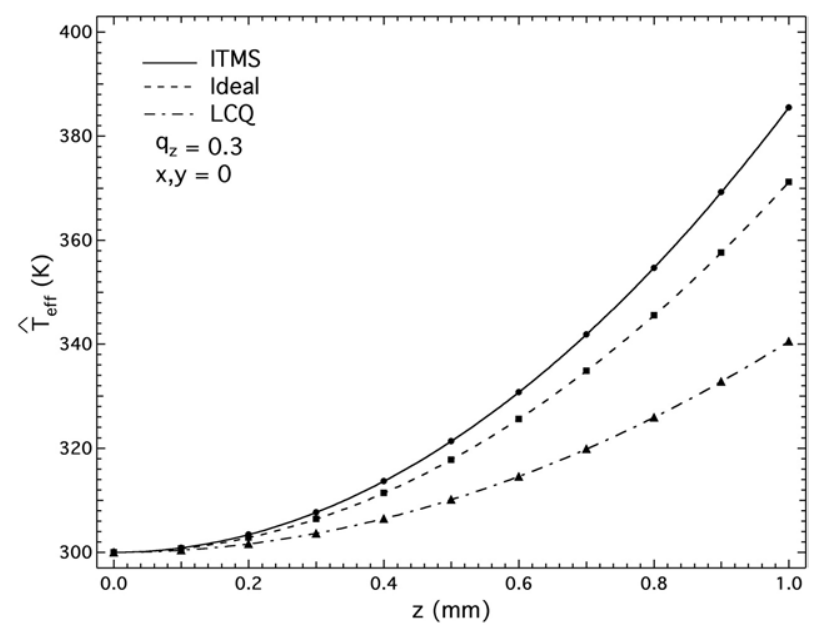

Figure 3. The time-averaged ion effective temperature, $\hat{T}_{\text {eff, }}$ at $q_{z}$ $=0.3$ for the Maxwell model of ion-neutral interactions calculated as a function of axial position, $z$, from the trap center. The individual curves represent results for three different ion trap configurations in which only the ring RF voltage is applied: ideal, ITMS, and LCQ. Each plotted point is the time average over 10 cycles of the fundamental axial frequency at $q_{z}=0.3$, after a delay time of $3 / \xi$ (about $4 \mathrm{~ms}$ for $\mathrm{m} / \mathrm{z} 100$ ions in He at $1 \mathrm{mTorr}$ ). 


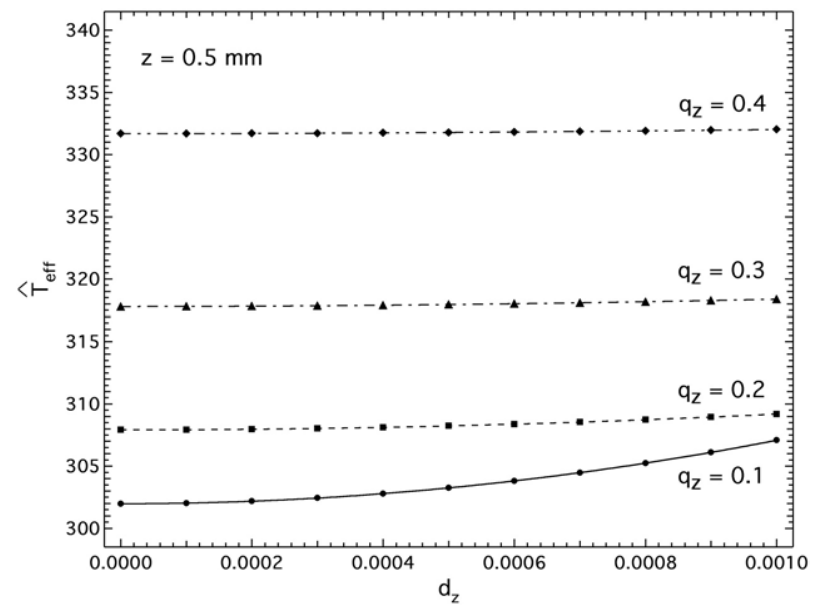

Figure 4. The time-averaged ion effective temperature, $\hat{T}_{e f f}$ at an axial distance $z=0.5 \mathrm{~mm}$ from the center of an ideal trap for the Maxwell model of ion-neutral interactions, calculated as a function of the parameterized dipolar excitation voltage, $d_{z}$ (see text). The peak-peak value of the dipolar excitation voltage, $V_{D}$, is linearly related to $d_{z}$, with $1023 \mathrm{mV}$ corresponding to $d_{z}=0.001$. The individual curves represent results for four different $q_{z}$ values. Each plotted point is the time average over 10 cycles of the fundamental axial frequency at $q_{z}=0.3$, after a delay time of $3 / \xi$ (about $4 \mathrm{~ms}$ for $\mathrm{m} / \mathrm{z} 100$ ions in He at $1 \mathrm{mTorr}$ ).

calculated as a function of $x$ or $y$ distance from the trap center at a fixed $q_{u=x, y, z}$ (not shown) followed a similar dependence to that in Figures 2 and 3. However, the increase in $\hat{T}_{\text {eff }}$ along those directions was $\sim 1 / 4$ that along the axial direction. The decrease in $\hat{T}_{\text {eff, }}$ ITMS $>$ Ideal > LCQ, seen in Figure 2 also is in the order and the approximate ratios expected from eq 24, since

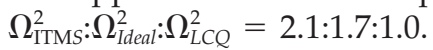

\section{Resonance Excitation}

As noted in the Introduction, resonance excitation is a widely used technique for increasing ion kinetic energy and, thus, $T_{\text {eff }}$ in ion traps. Figure 4 and Figure $5 \mathrm{a}$ and $\mathrm{b}$ are revealing in regard to the underlying means by which the increases in $T_{\text {eff }}$ occur. Figure 4 shows $\hat{T}_{\text {eff }}$ curves at $z=0.5 \mathrm{~mm}$ calculated for situations in which a dipolar excitation signal is applied to the endcaps of an ideal trap (in addition to application of the main trapping voltage to the ring). The plotted $\hat{T}_{\text {eff }}$ values represent the time average of $T_{\text {eff }}$ over 10 cycles of the appropriate $f_{z, 0}$, with a delay time of $\sim 4$ ms preceding each averaging step. The four curves correspond to selected $q_{z}$ values $(0.1,0.2,0.3,0.4)$; each curve covers a $d_{z}$ range from $0.000-0.0010$, which represents a peakpeak amplitude range of $0-1023 \mathrm{mV}$. For each $q_{z}$ curve, the frequency of the applied signal, $f_{z}=\omega_{z} / 2 \pi$ corresponds to the axial secular frequency, $f_{z, 0}$, determined from eq 30; that is, the dipolar signal is resonant with the ion secular oscillations. Note, however, that when $d_{z}$ $=0.000$ the situation corresponds to an ion trapping scenario, so any increase in $T_{\text {eff }}$ above thermal is due to ion RF heating only. Figure $5 \mathrm{a}$ and $\mathrm{b}$ show segments of the calculated time-dependent $T_{\text {eff }}$ data from which the $\hat{T}_{\text {eff }}$ data points at $\left[q_{z}=0.2,0.4 ; d_{z}=0.000,0.001\right]$ in Figure 4 were derived.

Over the indicated $d_{z}$ range in Figure 4 , the increase in $\hat{T}_{\text {eff }}$ due to dipolar excitation is small for any individual curve (e.g., only $1.3 \mathrm{~K}$ and $0.3 \mathrm{~K}$ for $d_{z}=0.001$ at $q_{z}$ $=0.2$ and 0.4 , respectively). In contrast, additional $\mathrm{RF}$ heating due to changes in $q_{z}\left(\propto V_{R}\right)$ results in significantly larger $\hat{T}_{\text {eff }}$ increases (e.g., $7.9 \mathrm{~K}$ and $31.7 \mathrm{~K}$ for $d_{z}=$ 0.000 at $q_{z}=0.2$ and 0.4 , respectively). These effects also can be seen in the time-dependent $T_{\text {eff }}$ plots in Figure $5 \mathrm{a}$ and $\mathrm{b}$. When $d_{z}=0.000$ the time-dependent $T_{\text {eff }}$ values vary at twice the main RF frequency, $2(\Omega / 2 \pi)$, since power is absorbed during both positive and negative phases of the main RF. Application of the dipolar excitation signal at $d_{z}=0.001$ results in the $2(\Omega / 2 \pi) T_{\text {eff }}$ oscilla-
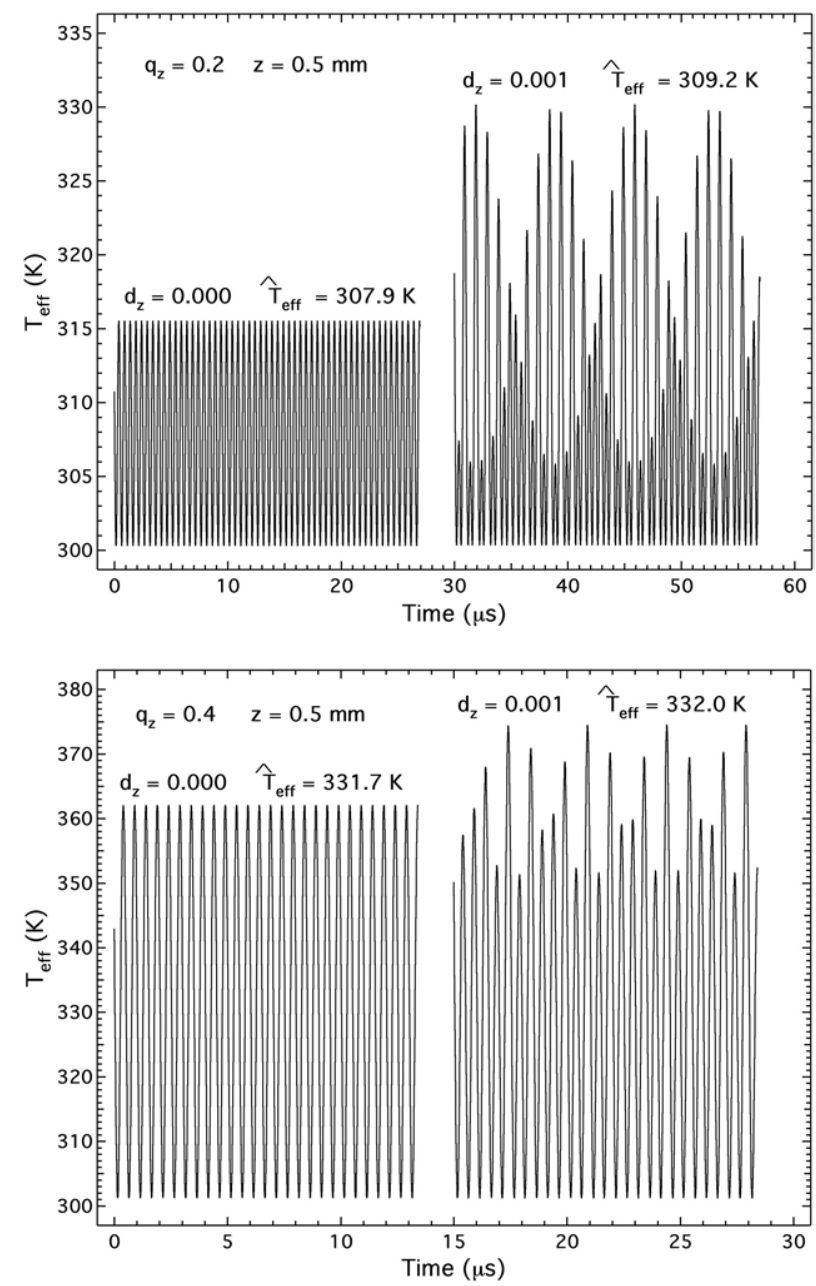

Figure 5. The time-dependent, ion effective temperature, $T_{\text {eff, }}$ at an axial distance of $z=0.5 \mathrm{~mm}$ from the trap center, calculated without $\left(d_{z}=0.000\right)$ and with $\left(d_{z}=0.001\right)$ a simulated dipolar excitation voltage applied to the endcaps. $\hat{T}_{\text {eff }}$ is the time average of $T_{\text {eff }}$ over four cycles of the fundamental axial frequency at the relevant $q_{z}$, after a delay time of $3 / \xi$ (about $4 \mathrm{~ms}$ for $m / z 100$ ions in He at 1 mTorr). (a) $q_{z}=0.2 ;(\mathbf{b}) q_{z}=0.4$. 
tions being modulated at $2 f_{z}=2\left(\frac{\omega}{2 \pi}\right)$. Although the depth of modulation is greater for $q_{z}=0.2$ than for $q_{z}=0.4$ (because $\left.V_{R}\left(q_{z}=0.2\right)<V_{R}\left(q_{z}=0.4\right)\right)$, in each case it is considerably less than the amplitude of oscillations at $2(\Omega / 2 \pi)$. These results suggest that ion heating in the resonance excitation process is primarily due to increased power absorption from the RF field rather than from the dipolar excitation signal. The dipolar excitation signal mainly serves to move ions into regions of the ion trap where the RF electric field, and thus ion RF heating, is greater than near the trap center.

\section{Conclusions}

The moment equations resulting from transformation of the Boltzmann equation retain a significant attribute: the temporal and spatial dependence of the ensemble average value for any property that is a function of the ion velocity can be determined from the corresponding moment. It is possible to apply moment theory to RF ion traps because spatial gradients of the ion number density are much smaller than spatial variations of the electric field and typical momentum-transfer collision frequencies are much smaller than the RF frequencies involved. In this paper, both ideal and non-ideal ion trap configurations are accounted for in the two-temperature moment equations by incorporating parameterized variables $\widetilde{a}$ and $\widetilde{q}$, which are modified versions of the commonly used forms for the DC and AC ring voltages, and $\tilde{b}$ and $\tilde{d}$, which are new forms that account for the voltages applied to the endcaps.

Application of the moment equations to specific situations has been focused on the space and time dependence of the ion effective temperature during ion storage and resonance excitation. Calculations suggest that increases in effective temperature during resonance excitation are due primarily to power absorption from the main RF trapping field rather than from the dipolar excitation signal. The dipolar excitation signal apparently serves mainly to move ions into regions of the ion trap where the RF electric field, and thus ion RF heating, is greater than near the trap center. The applicability, however, is not limited to just ion storage and resonance excitation processes. For example, application of the above moment equations to the boundary activation technique to determine effective temperature behavior for that method also should be straightforward. Of major interest as well is the dynamic behavior of the average effective temperature for the entire trapped ion population. Obtaining that information requires using the ion number density distribution, $n(x, y, z, t)$, to weight the $T_{\text {eff }}$ values at each position by the corresponding number of ions, summing over all positions, and averaging over all ions. An expression for $n(x, y, z, t)$ has been obtained elsewhere by solving the equation of continuity [49]. That result, however, does not consider the effect of the space charge field induced by the trapped ion population, a process that is outside the scope of this paper.

Besides extending the applicability of the moment equations to non-ideal quadrupole ion traps, the modified versions of the parameterized variables can have additional utility. For example, calculation of the spatial dependence of ion secular oscillation frequencies for different ion trap configurations is possible. Such calculations might potentially be used at the design stage for new ion trap configurations to reduce expected chemical mass shifts [56]. On the other hand, the moment equations also are subject to limitations of the Boltzmann equation from which they are derived; the significance to this work is that applicable systems are restricted to those comprised of trace concentrations of atomic ions moving through a pure, dilute buffer gas consisting of unreactive atomic neutrals. The latter limitation will be addressed in a forthcoming paper that extends ion trap moment theory to molecular systems.

\section{Acknowledgments}

The work carried out by DEG was sponsored by the Office of Basic Energy Sciences, U.S. Department of Energy. Oak Ridge National Laboratory is managed by UT-Battelle, LLC, for the U.S. Department of Energy, under contract no. DE-AC05-00OR22725. The research of LAV and DMD was supported by a National Science Foundation grant. The authors also thank the anonymous reviewers for helpful suggestions with regard to manuscript modifications.

This manuscript has been authored by a contractor of the U.S. Government under contract no. DE-AC05-00OR22725. Accordingly, the U.S. Government retains a paid-up, nonexclusive, irrevocable, worldwide license to publish or reproduce the published form of this contribution, prepare derivative works, distribute copies to the public, and perform publicly and display publicly, or allow others to do so, for U.S. Government purposes.

\section{References}

1. Wiley, W. C.; McLaren, I. H. Time-of-Flight Mass Spectrometer with Improved Resolution. Rev. Sci. Instrum. 1955, 26, 1150.

2. Paul, W.; Steinwedel, H. A New Mass Spectrometer Without a Magnetic Field. Z. Naturforsch 1953, 8a, 448-450.

3. Gerlich, D. Inhomogeneous Rf-Fields-A Versatile Tool for the Study of Processes with Slow Ions. Adv. Chem. Phys. 1992, 82, 1-176.

4. Dawson, P. H. Quadrupole Mass Spectrometry and Its Applications; Elsevier: Amsterdam, 1976

5. March, R. E.; Hughes, R. J. Quadrupole Storage Mass Spectrometry; Wiley-Interscience: New York, 1989.

6. Michael, S. M.; Chien, M.; Lubman, D. M. An Ion Trap Storage Time-of-Flight Mass-Spectrometer. Rev. Sci. Instrum. 1992, 63, 42774284

7. Campbell, J. M.; Collings, B. A.; Douglas, D. J. A New Linear Ion Trap Time-of-Flight System with Tandem Mass Spectrometry Capabilities. Rapid Commun. Mass Spectrom. 1998, 12, 1463-1474.

8. Hager, J. W. Recent Trends in Mass Spectrometer Development. Anal. Bioanal. Chem. 2004, 378, 845-850.

9. Douglas, D. J.; Frank, A. J.; Mao, D. M. Linear Ion Traps in Mass Spectrometry. Mass Spectrom. Rev. 2005, 24, 1-29.

10. Franzen, J.; Gabling, R. H.; Schubert, M.; Wang, Y., Nonlinear ion traps. In Practical Aspects of Ion Trap Mass Spectrometry; Vol. I; March, R. E.; Todd, J. F. J., Eds.; CRC Press: New York, 1995; pp 49-167.

11. Hager, J. W. A New Linear Ion Trap Mass Spectrometer. Rapid Commun. Mass Spectrom. 2002, 16, 512-526.

12. Stafford, G. C.; Kelley, P. E.; Syka, J. E. P.; Reynolds, W. E.; Todd, J. F. J. Recent Improvements in and Analytical Applications of Advanced Ion Trap Technology. Int. J. Mass Spectrom. Ion Processes 1984, 60, 85-98.

13. Douglas, D. J.; French, J. B. Collisional Focusing Effects in Radiofrequency Quadrupoles. J. Am. Soc. Mass Spectrom 1992, 3, 398-408.

14. Tolmachev, A. V.; Chernushevich, I. V.; Dodonov, A. F.; Standing, K. G. A Collisional Focusing Ion Guide for Coupling an Atmospheric Pres- 
sure Ion Source to a Mass Spectrometer. Beam Interactions with Materials and Atoms. Nucl. Instrum. Methods Phys. Res. B 1997, 124, 112-119.

15. Louris, J. N.; Cooks, R. G.; Syka, J. E. P.; Kelley, P. E.; Stafford, G. C., Jr.; Todd, J. F. J. Instrumentation, Applications, and Energy Deposition in Quadrupole Ion-Trap Tandem Mass Spectrometry. Anal. Chem. 1987, 59, 1677-1685.

16. McLuckey, S. A.; Goeringer, D. E. Slow Heating Methods in Tandem Mass Spectrometry. J. Mass Spectrom. 1997, 32, 461-474.

17. Blatt, R.; Zoller, P.; Holzmueller, G.; Siemers, I. Brownian Motion of a Parametric Oscillator: A Model for Ion Confinement in Radio Frequency Traps. Z. Phys. D. At. Mol. Clusters 1986, 4, 121-126.

18. Moriwaki, Y.; Tachikawa, M.; Maeno, Y.; Shimizu, T. Laser Spectroscopic Study of Dynamics of Laser-Cooled Ions in Paul Trap. Proceedings of the AIP Conference; 1994; pp 38-39.

19. Moriwaki, Y.; Tachikawa, M.; Maeno, Y.; Shimizu, T. Collision Cooling of Ions Stored in Quadrupole Radiofrequency Trap. Japn. J. Applied Phys. Part 2 Lett. 1992, 31, L1640-L1643.

20. Vedel, F.; Andre, J.; Vedel, M.; Brincourt, G. Computed Energy and Spatial Statistical Properties of Stored Ions Cooled by a Buffer Gas. Phys. Rev. A. 1983, 27, 2321-2330.

21. Baranov, V. Ion Energy in Quadrupole Mass Spectrometry. J. Am. Soc. Mass Spectrom. 2004, 15, 48-54.

22. Wang, Y. Nonlinear Resonance Conditions and Their Relationship to Higher Multipole Fields in Ion Traps and Quadrupole Mass Filters. Rapid Commun. Mass Spectrom. 1993, 7, 920-928.

23. Lunney, M. D. N.; Webb, J. P.; Moore, R. B. Finite-Element Analysis of Radio Frequency Quadrupole Traps. J. Appl. Phys. 1989, 65, 2883-2888.

24. Londry, F. A.; Alfred, R. L.; March, R. E. Computer-Simulation of Single-Ion Trajectories in Paul-Type Ion Traps. J. Am. Soc. Mass Spectrom. 1993, 4, 687-705.

25. Julian, R. K.; Reiser, H. P.; Cooks, R. G. Large-Scale Simulation of Mass-Spectra Recorded with a Quadrupole Ion Trap Mass-Spectrometer. Int. J. Mass Spectrom. Ion Processes 1993, 123, 85-96.

26. Reiser, H. P.; Julian, R. K.; Cooks, R. G. A Versatile Method of Simulation of the Operation of Ion Trap Mass Spectrometers. Int. J. Mass Spectrom. Ion Processes 1992, 121, 49-63.

27. Parks, J. H.; Szoke, A. Simulation of Collisional Relaxation of Trapped Ion Clouds in the Presence of Space Charge Fields. J. Chem. Phys. 1995, 103, 1422-1439.

28. Dawson, P. H.; Whetten, N. R. Quadrupoles, Monopoles, and Ion Traps. Res. Develop. 1968, 19, 46-50.

29. Bonner, R. F.; March, R. E.; Durup, J. Effect of Charge Exchange Reactions on the Motion of Ions in Three-Dimensional Quadrupole Electric Fields. Int. J. Mass Spectrom. Ion Phys. 1976, 22, 17-34.

30. Viehland, L. A.; Mason, E. A. Gaseous Ion Mobility in Electric-Fields of Arbitrary Strength. Ann. Phys. 1975, 91, 499-533.

31. Viehland, L. A.; Mason, E. A. Gaseous Ion Mobility and Diffusion in Electric-Fields of Arbitrary Strength. Ann. Phys. 1978, 110, 287-328.

32. Vekey, K. Internal Energy Effects in Mass Spectrometry. J. Mass Spectrom. 1996, 31, 445-463.

33. Drahos, L.; Vekey, K. Special Feature: Commentary-How Closely Related are the Effective and the Real Temperature. J. Mass Spectrom. 1999, 34, 79-84.
34. McLuckey, S. A.; Cameron, D.; Cooks, R. G. Proton Affinities from Dissociations of Proton-Bound Dimers. . Am. Chem. Soc. 1981, 103, 1313-1317.

35. Goeringer, D. E. McLuckey, S. A. Evolution of Ion Internal Energy During Collisional Excitation in the Paul Ion Trap : A Stochastic Approach. J. Chem. Phys. 1996, 104, 2214-2221.

36. Goeringer, D. E.; McLuckey, S. A. Kinetics of Collision-Induced Dissociation in the Paul Trap: A First-Order Model. Rapid Commun. Mass Spectrom 1996, 10, 328-334.

37. Lin, S. L.; Bardsley, J. N. Monte-Carlo Simulation of Ion Motion in Drift Tubes. J. Chem. Phys. 1977, 66, 435-445.

38. Skullerud, H. R. Monte-Carlo Investigations of Motion of Gaseous Ions in Electrostatic Fields. J. Phys B At. Mol. Opt. Phys. 1973, 6, 728-742.

39. Plass, W. R.; Gill, L. A.; Bui, H. A.; Cooks, R. G. Ion Mobility Measurement by dc Tomography in an RF Quadrupole Ion Trap. J. Phys. Chem. A 2000, 104, 5059-5065.

40. Viehland, L. A.; Mason, E. A.; Whealton, J. H. Kinetic Theory of Ion-Cyclotron Resonance Collision Broadening. J. Chem. Phys. 1975, 62, 4715-4726.

41. Viehland, L. A.; Kabbe, E. A.; Dixit, V. V. Moment Theory of Ion Motion in Traps and Similar Devices: II. Cylindrical Apparatus. J. Phys. B At. Mol. Opt. Phys. 2005, 38, 4011-4026.

42. Viehland, L. A.; Goeringer, D. E. Kinetic Theory of Radio Frequency Quadrupole Ion Traps. I. Trapping of Atomic Ions in a Pure Atomic Gas. J. Chem. Phys. 2004, 120, 9090-9103.

43. Cercignani, C. The Boltzmann Equation and Its Applications; SpringerVerlag: New York, 1988.

44. Mason, E. A.; McDaniel, E. W. Transport Properties of Ions in Gases; Wiley-Interscience: New York, 1988, p. 193.

45. Viehland, L. A.; Mason, E. A. Gaseous Ion Mobility in Electric Fields of Arbitrary Strength. Ann. Phys. 1975, 91, 499-533.

46. Lin, S. L.; Viehland, L. A.; Mason, E. A. Three-Temperature Theory of Gaseous Ion-Transport. Chem. Phys. 1979, 37, 411-424.

47. Viehland, L. A.; Lin, S. L. Application of the Three-Temperature Theory of Gaseous Ion-Transport. Chem. Phys. 1979, 43, 135-144.

48. Viehland, L. A.; Goeringer, D. E. Moment Theory of Ion Motion in Traps and Similar Devices: I. General Theories. J. Phys. B At. Mol. Opt. Phys. 2005, 38, 3987-4009.

49. Goeringer, D. E.; Viehland, L. A. Moment Theory of Ion Motion in Traps and Similar Devices: III. Two-Temperature Treatment of Quadrupole Ion Traps. J. Phys. B At. Mol. Opt. Phys. 2005, 38, 4027-4044.

50. Wannier, G. H. Motion of Gaseous Ions in Strong Electric Fields. Bell Syst. Tech. J. 1953, 32, 170-254

51. Ferziger, J. H.; Kaper, H. G. Mathematical Theory of Transport Processes in Gases; North-Holland: Amsterdam, 1972, p. 24.

52. Practical Aspects of Ion Trap Mass Spectrometry, Vol. I : Fundamentals of Ion Trap Mass Spectrometry; CRC Press: Boca Raton, FL, 1995, p. 144.

53. McDaniel, E. W.; Mason, E. A. The Mobility and Diffusion of Ions in Gases; John Wiley and Sons: New York, 1973, p. 236.

54. Viehland, L. A. Gaseous Ion-Transport Coefficients. Chem. Phys. 1982, 70, 149-156.

55. Viehland, L. A. Interaction Potentials for the Alkali Ion Rare-Gas Systems. Chem. Phys. 1984, 85, 291-305.

56. Plass, W. R.; Li, H. Y.; Cooks, R. G. Theory, Simulation, and Measurement of Chemical Mass Shifts in RF Quadrupole Ion Traps. Int. J. Mass Spectrom. 2003, 228, 237-267.

\section{Appendix A}

\section{Parameterized Variables for Non-Ideal Traps}

In eqs $23 a$ and $b, 24,25 a$ and $b$, the $D C$ and $R F$ ring electrode voltages have been converted into the dimensionless, parameterized variables $\tilde{a}$ and $\tilde{q}$ that are defined by eqs A.1a and $\mathrm{b}$. The additional variables $\tilde{b}$ and $\tilde{d}$, defined by eqs A.2a and b, are parameterized forms of the voltages applied to the endcaps. The forms of $\tilde{a}$ and $\tilde{q}$ are similar to the parameterized variables $a$ and $b$ (see eqs 19a and b) commonly used in ion trap studies, but they are modified by terms that include the expansion coefficients, $A_{l}$, to take into account a specific configuration. Similarly, $\tilde{b}$ and $\tilde{d}$ are modified forms of the parameterized variables $b$ and $d$ (see eqs $21 \mathrm{a}$ and $\mathrm{b}$ ). The ellipses in eqs A.1a-A.2b) represent higher order terms that could be obtained from eq 22 if necessary.

$$
\frac{\widetilde{a}_{u}}{a_{u}}=\frac{\widetilde{q}_{u}}{q_{u}}=\frac{-A_{2}^{R}\left(r_{0}^{2}+2 z_{0}^{2}\right)}{2 r_{0}^{2}}\left[1+\frac{A_{4}^{R}}{A_{2}^{R}} \frac{12 z^{2}-3\left(x^{2}+y^{2}\right)}{2 r_{0}^{2}}+\frac{A_{6}^{R}}{A_{2}^{R}} \frac{120 z^{4}-180 z^{2}\left(x^{2}+y^{2}\right)+15\left(x^{2}+y^{2}\right)^{2}}{8 r_{0}^{4}}+\ldots\right] u=x, y
$$

$$
\frac{\widetilde{a}_{z}}{a_{z}}=\frac{\widetilde{q}_{z}}{q_{z}}=\frac{-A_{2}^{R}\left(r_{0}^{2}+2 z_{0}^{2}\right)}{2 r_{0}^{2}}\left[1+\frac{A_{4}^{R}}{A_{2}^{R}} \frac{2 z^{2}-3\left(x^{2}+y^{2}\right)}{r_{0}^{2}}+\frac{A_{6}^{R}}{A_{2}^{R}} \frac{24 z^{4}-120 z^{2}\left(x^{2}+y^{2}\right)+45\left(x^{2}+y^{2}\right)^{2}}{8 r_{0}^{4}}+\ldots\right]
$$




$$
\begin{aligned}
& \frac{\tilde{b}_{u}}{b_{u}}=\frac{\tilde{d}_{u}}{d_{u}}=\frac{A_{1}^{D}\left(r_{0}^{2}+2 z_{0}^{2}\right)}{4 r_{0}^{2}}\left[0+6 \frac{A_{3}^{D}}{A_{1}^{D}}+\frac{A_{5}^{D}}{A_{1}^{D}} \frac{20 z^{2}-15\left(x^{2}+y^{2}\right)}{r_{0}^{2}}+\ldots\right] u=x, y \\
& \frac{\tilde{b}_{z}}{b_{z}}=\frac{\tilde{d}_{z}}{d_{z}}=\frac{A_{1}^{D}\left(r_{0}^{2}+2 z_{0}^{2}\right)}{4 r_{0}^{2}}\left[1+\frac{A_{3}^{D}}{A_{1}^{D}} \frac{6 z^{2}-3\left(x^{2}+y^{2}\right)}{2 r_{0}^{2}}+\frac{A_{5}^{D}}{A_{1}^{D}} \frac{40 z^{4}-120 z^{2}\left(x^{2}+y^{2}\right)+15\left(x^{2}+y^{2}\right)^{2}}{8 r_{0}^{4}}+\ldots\right]
\end{aligned}
$$

\section{Appendix B}

\section{Solutions of Moment Equations for Non-Ideal Traps with Maxwell Collisions}

For the Maxwell model in which $\xi$ is constant, the solution of eqs $25 \mathrm{a}$ and $\mathrm{b}$ for the average ion velocity component, $\overline{\mathrm{v}_{u}}(t)$, is given in eq B.2, with $\tilde{f}_{u}$ defined for all $u$ in eq B.1.

$$
\begin{aligned}
& \tilde{f}_{u}=\left[\begin{array}{cl}
\frac{z u}{r_{0}} & u=x, y \\
r_{0} & u=z
\end{array}\right. \\
& \overline{\mathrm{v}}_{u}(t)= \overline{\mathrm{v}_{u}}(0) \exp (-\xi t)-\frac{\Omega^{2}}{4 \xi}\left[\widetilde{a}_{u} u+\tilde{b}_{u} \tilde{f}_{u}\right][1-\exp (-\xi t)]+\widetilde{q}_{u} u \frac{\Omega^{2}}{2\left(\xi^{2}+\Omega^{2}\right)}[\xi \cos (\Omega t) \\
&+\Omega \sin (\Omega t)-\xi \exp (-\xi t)]+\widetilde{d}_{u} \tilde{f}_{u} \frac{\Omega^{2} \cos \left(\delta_{D}\right)}{2\left(\xi^{2}+\omega_{D}^{2}\right)}\left[\xi \cos \left(\omega_{D} t\right)+\omega_{D} \sin \left(\omega_{D} t\right)-\xi \exp (-\xi t)\right] \\
&+\tilde{d}_{u} \tilde{f}_{u} \frac{\Omega^{2} \sin \left(\delta_{D}\right)}{2\left(\xi^{2}+\omega_{D}^{2}\right)}\left[\omega_{D} \cos \left(\omega_{D} t\right)-\xi \sin \left(\omega_{D} t\right)-\omega_{D} \exp (-\xi t)\right]
\end{aligned}
$$

The dimensionless temperature ratio, $\varepsilon=\frac{T_{\text {eff }}}{T}$, for the Maxwell model is obtained by substituting the results for $\overline{v_{u}}$ given above by eq B.2, into eq 24 . The solution of the resulting first-order differential equation is facilitated by first writing it as eq B.3

$$
\begin{aligned}
\frac{d}{\mathrm{~d} t} \varepsilon & +\widetilde{A} \exp (-\xi t)+\widetilde{B}_{1} \cos (\Omega t) \exp (-\xi t)+\widetilde{B}_{2} \cos \left(\omega_{D} t+\delta\right) \exp (-\xi t)+\widetilde{C}_{1} \cos (\Omega t) \\
& +\widetilde{C}_{2} \cos \left(\omega_{D} t+\delta\right)+\widetilde{D}_{1} \sin (\Omega t)+\widetilde{D}_{2} \sin \left(\omega_{D} t+\delta\right)+\widetilde{E}_{1} \cos ^{2}(\Omega t)+\widetilde{E}_{2} \cos (\Omega t) \cos \left(\omega_{D} t+\delta\right) \\
& +\widetilde{E}_{3} \cos ^{2}\left(\omega_{D} t+\delta\right)+\widetilde{F}_{1} \cos (\Omega t) \sin (\Omega t)+\widetilde{F}_{2} \cos (\Omega t) \sin \left(\omega_{D} t+\delta\right)+\widetilde{F}_{3} \sin (\Omega t) \cos \left(\omega_{D} t+\delta\right) \\
& +\widetilde{F}_{4} \sin \left(\omega_{D} t+\delta\right) \cos \left(\omega_{D} t+\delta\right)+\widetilde{G}+v \varepsilon=0,
\end{aligned}
$$

where

$$
\begin{aligned}
& \widetilde{A}=\frac{\mu \Omega^{2}}{6 k_{B} T} \sum_{u}\left(\widetilde{a}_{u} u+\tilde{b}_{u} \tilde{f}_{u}\right)\left[\overline{\mathrm{v}_{u}}(0)+\frac{\Omega^{2}}{4 \xi}\left(\widetilde{a}_{u} u+\tilde{b}_{u} \tilde{f}_{u}\right)-\frac{\Omega^{2} \xi}{2\left(\xi^{2}+\Omega^{2}\right)} \widetilde{q}_{u} u-\frac{\Omega^{2} \xi}{2\left(\xi^{2}+\omega_{D}^{2}\right)} \widetilde{d}_{u} \tilde{f}_{u} \cos (\delta)-\frac{\Omega^{2} \omega_{D}}{2\left(\xi^{2}+\omega_{D}^{2}\right)} \widetilde{d}_{u} \tilde{f}_{u} \sin (\delta)\right. \\
& \widetilde{B}_{1}=\frac{-\mu \Omega^{2}}{3 k_{B} T} \sum_{u} \widetilde{q}_{u} u\left[\overline{\mathrm{v}_{u}}(0)+\frac{\Omega^{2}}{4 \xi}\left(\widetilde{a}_{u} u+\tilde{b}_{u} \tilde{f}_{u}\right)-\frac{\Omega^{2} \xi}{2\left(\xi^{2}+\Omega^{2}\right)} \widetilde{q}_{u} u-\frac{\Omega^{2} \xi}{2\left(\xi^{2}+\omega_{D}^{2}\right)} \widetilde{d}_{u} \tilde{f}_{u} \cos (\delta)-\frac{\Omega^{2} \omega_{D}}{2\left(\xi^{2}+\omega_{D}^{2}\right)} \widetilde{d}_{u} \tilde{f}_{u} \sin (\delta)\right] \\
& \widetilde{B}_{2}=\frac{-\mu \Omega^{2}}{3 k_{B} T} \sum_{u} \tilde{d}_{u} \tilde{f} u\left[\overline{\mathrm{v}}_{\mathbf{u}}(0)+\frac{\Omega^{2}}{4 \xi}\left(\widetilde{a}_{u} u+\tilde{b}_{u} \tilde{f}_{u}\right)-\frac{\Omega^{2} \xi}{2\left(\xi^{2}+\Omega^{2}\right)} \widetilde{q}_{u} u-\frac{\Omega^{2} \xi}{2\left(\xi^{2}+\omega_{D}^{2}\right)} \tilde{d}_{u} \tilde{f}_{u} \cos (\delta)-\frac{\Omega^{2} \omega_{D}}{2\left(\xi^{2}+\omega_{D}^{2}\right)} \tilde{d}_{u} \tilde{f}_{u} \sin (\delta)\right] \\
& \widetilde{C}_{1}=\frac{\mu \Omega^{4}}{12 k_{B} T \xi} \frac{2 \xi^{2}+\Omega^{2}}{\xi^{2}+\Omega^{2}} \sum_{u}\left(\widetilde{a}_{u} u+\tilde{b}_{u} \tilde{f}_{u}\right) \tilde{q}_{u} u \quad \widetilde{C}_{2}=\frac{\mu \Omega^{4}}{12 k_{B} T \xi} \frac{2 \xi^{2}+\omega_{D}^{2}}{\xi^{2}+\omega_{D}^{2}} \sum_{u}\left(\widetilde{a}_{u} u+\tilde{b}_{u} \tilde{f}_{u}\right) \tilde{d}_{u} \tilde{f}_{u}
\end{aligned}
$$




$$
\begin{aligned}
& \widetilde{D}_{1}=\frac{\mu \Omega^{4}}{12 k_{B} T} \frac{\Omega}{\xi^{2}+\Omega^{2}} \sum_{u}\left(\widetilde{a}_{u} u+\widetilde{b}_{u} \tilde{f}_{u}\right) \widetilde{q}_{u} u \quad \widetilde{D}_{2}=\frac{\mu \Omega^{4}}{12 k_{B} T} \frac{\omega_{D}}{\xi^{2}+\omega_{D}^{2}} \sum_{u}\left(\widetilde{a}_{u} u+\tilde{b}_{u} \tilde{f}_{u}\right) \tilde{d}_{u} \tilde{f}_{u} \\
& \widetilde{E}_{1}=\frac{-\mu \Omega^{4}}{6 k_{B} T} \frac{\xi}{\xi^{2}+\Omega^{2}} \sum_{u} \widetilde{q}_{u}^{2} u^{2} \quad \widetilde{E}_{2}=\frac{-\mu \Omega^{4}}{6 k_{B} T}\left(\frac{\xi}{\xi^{2}+\Omega^{2}}+\frac{\xi}{\xi^{2}+\omega_{D}^{2}}\right) \sum_{u} \widetilde{q}_{u} u \widetilde{d}_{u} \tilde{f}_{u} \\
& \widetilde{E}_{3}=\frac{-\mu \Omega^{4}}{6 k_{B} T} \frac{\xi}{\xi^{2}+\omega_{D}^{2}} \sum_{u} \widetilde{d}_{u}^{2} \tilde{f}_{u}^{2} \\
& \widetilde{F}_{1}=\frac{-\mu \Omega^{4}}{6 k_{B} T} \frac{\Omega}{\xi^{2}+\Omega^{2}} \sum_{u} \widetilde{q}_{u}^{2} u^{2} \quad \widetilde{F}_{2}=\frac{-\mu \Omega^{4}}{6 k_{B} T}\left(\frac{\omega_{D}}{\xi^{2}+\omega_{D}^{2}}\right) \sum_{u} \widetilde{q}_{u} u \widetilde{d}_{u} \tilde{f}_{u} \\
& \widetilde{F}_{3}=\frac{-\mu \Omega^{4}}{6 k_{B} T}\left(\frac{\Omega}{\xi^{2}+\Omega^{2}}\right) \sum_{u} \widetilde{q}_{u} u \widetilde{d}_{u} \tilde{f}_{u} \quad \widetilde{F}_{4}=\frac{-\mu \Omega^{4}}{6 k_{B} T}\left(\frac{\omega_{D}}{\xi^{2}+\omega_{D}^{2}}\right) \sum_{u} \widetilde{d}_{u}^{2} \tilde{f}_{u}^{2} \\
& \widetilde{G}=\frac{-\mu \Omega^{4}}{24 k_{B} T \xi} \sum_{u}\left(\widetilde{a}_{u} u+\widetilde{b}_{u} \tilde{f}_{u}\right)^{2}-\nu \quad \nu=\frac{2 m \xi}{m+M}
\end{aligned}
$$

Then it is straightforward but extraordinarily tedious to find by the use of Laplace transforms that

$$
\begin{aligned}
\varepsilon(t)= & \varepsilon_{0} \exp (-\nu t)-\frac{\widetilde{A}}{\nu-\xi}[\exp (-\xi t)-\exp (-\nu t)] \\
& -\frac{\widetilde{B_{1}}}{(\nu-\xi)^{2}+\Omega^{2}}[(\nu-\xi)\{\cos (\Omega t) \exp (-\xi t)-\exp (-\nu t)\}+\Omega \sin (\Omega t) \exp (-\xi t)] \\
& -\frac{\widetilde{B_{2}}}{(\nu-\xi)^{2}+\omega_{D}^{2}}\left[\left\{(\nu-\xi) \cos (\delta)+\omega_{D} \sin (\delta)\right\}\left\{\cos \left(\omega_{D} t\right) \exp (-\xi t)-\exp (-\nu t)\right\}\right. \\
& \left.+\left\{\omega_{D} \cos (\delta)-(\nu-\xi) \sin (\delta)\right\} \sin \left(\omega_{D} t\right) \exp (-\xi t)\right]-\frac{\widetilde{C}_{1}}{\nu^{2}+\Omega^{2}}[\nu\{\cos (\Omega t)-\exp (-\nu t)\}+\Omega \sin (\Omega t)] \\
& -\frac{\widetilde{C_{2}}}{\nu^{2}+\omega_{D}^{2}}\left[\left\{\nu \cos (\delta)+\omega_{D} \sin (\delta)\right\}\left\{\cos \left(\omega_{D} t\right)-\exp (-\nu t)\right\}+\left\{\omega_{D} \cos (\delta)-\nu \sin (\delta)\right\} \sin \left(\omega_{D} t\right)\right] \\
& +\frac{\widetilde{D}_{1}}{\nu^{2}+\Omega^{2}}[\Omega\{\cos (\Omega t)-\exp (-\nu t)\}-\nu \sin (\Omega t)] \\
& +\frac{\widetilde{D_{2}}}{\nu^{2}+\omega_{D}^{2}}\left[\left\{\omega_{D} \cos (\delta)-\nu \sin (\delta)\right\}\left\{\cos \left(\omega_{D} t\right)-\exp (-\nu t)\right\}-\left\{\nu \cos (\delta)+\omega_{D} \sin (\delta)\right\} \sin \left(\omega_{D} t\right)\right] \\
& \left.+\left\{\left(\omega_{D}-\Omega\right) \cos (\delta)-\nu \sin (\delta)\right\} \sin \left(\left(\omega_{D}-\Omega\right) t\right)\right] \\
& -\frac{\widetilde{E_{1}}}{2 \nu}[1-\exp (-\nu t)]-\frac{\widetilde{E_{1}}}{2\left(\nu^{2}+4 \Omega^{2}\right)}[\nu\{\cos (2 \Omega t)-\exp (-\nu t)\}+2 \Omega \sin (2 \Omega t)] \\
& \frac{\widetilde{E_{2}}}{2\left(\nu^{2}+\left(\omega_{D}-\Omega\right)^{2}\right)}\left[\left\{\nu \cos (\delta)+\left(\omega_{D}-\Omega\right) \sin (\delta)\right\}\left\{\cos \left(\left(\omega_{D}-\Omega\right) t\right)-\exp (-\nu t)\right\}\right. \\
& \\
&
\end{aligned}
$$




$$
\begin{aligned}
& -\frac{\widetilde{E}_{2}}{2\left(\nu^{2}+\left(\omega_{D}+\Omega\right)^{2}\right)}\left[\left\{\nu \cos (\delta)+\left(\omega_{D}+\Omega\right) \sin (\delta)\right\}\left\{\cos \left(\left(\omega_{D}+\Omega\right) t\right)-\exp (-\nu t)\right\}\right. \\
& \left.+\left\{\left(\omega_{D}+\Omega\right) \cos (\delta)-\nu \sin (\delta)\right\} \sin \left(\left(\omega_{D}+\Omega\right) t\right)\right]-\frac{\widetilde{E}_{3}}{2 \nu}[1-\exp (-\nu t)] \\
& -\frac{\widetilde{E}_{3}}{2\left(\nu^{2}+4 \omega_{D}^{2}\right)}\left[\left\{\nu \cos ^{2}(\delta)+4 \omega_{D} \sin (\delta) \cos (\delta)-\nu \sin ^{2}(\delta)\right\}\left\{\cos \left(2 \omega_{D} t\right)-\exp (-\nu t)\right\}\right. \\
& \left.+2\left\{\omega_{D} \cos ^{2}(\delta)-\nu \sin (\delta) \cos (\delta)-\omega_{D} \sin ^{2}(\delta)\right\} \sin \left(2 \omega_{D} t\right)\right] \\
& -\frac{\widetilde{F}_{1}}{2\left(\nu^{2}+4 \Omega^{2}\right)}[2 \Omega\{\cos (2 \Omega t)-\exp (-\nu t)\}-\nu \sin (2 \Omega t)] \\
& +\frac{\widetilde{F}_{2}+\widetilde{F}_{3}}{2\left(\nu^{2}+\left(\omega_{D}+\Omega\right)^{2}\right)}\left[\left\{\left(\omega_{D}+\Omega\right) \cos (\delta)-\nu \sin (\delta)\right\}\left\{\cos \left(\left(\omega_{D}+\Omega\right) t\right)-\exp (-\nu t)\right\}\right. \\
& \left.-\left\{\nu \cos (\delta)+\left(\omega_{D}+\Omega\right) \sin (\delta)\right\} \sin \left(\left(\omega_{D}+\Omega\right) t\right)\right] \\
& +\frac{\widetilde{F}_{2}+\widetilde{F}_{3}}{2\left(\nu^{2}+\left(\omega_{D}-\Omega\right)^{2}\right)}\left[\left\{\left(\omega_{D}-\Omega\right) \cos (\delta)-\nu \sin (\delta)\right\} \cos \left(\left(\omega_{D}-\Omega\right) t\right)-\exp (-\nu t)\right\} \\
& \left.-\left\{\nu \cos (\delta)+\left(\omega_{D}-\Omega\right) \sin (\delta)\right\} \sin \left(\left(\omega_{D}-\Omega\right) t\right)\right] \\
& +\frac{\widetilde{F}_{4}}{2\left(\nu^{2}+4 \omega_{D}^{2}\right)}\left[2\left\{\omega_{D} \cos ^{2}(\delta)-\nu \sin (\delta) \cos (\delta)-\omega_{D} \sin ^{2}(\delta)\right\}\left\{\cos \left(2 \omega_{D} t\right)-\exp (-\nu t)\right\}\right. \\
& \left.-\left\{\nu \cos ^{2}(\delta)+4 \omega_{D} \sin (\delta) \cos (\delta)-\nu \sin ^{2}(\delta)\right\} \sin \left(2 \omega_{D} t\right)\right]-\frac{\widetilde{G}}{\nu}[1-\exp (-\nu t)]
\end{aligned}
$$

It should be noted that the dependence of $\varepsilon$ upon time is explicit in this result, but its dependence upon position is implicit through its dependence upon the quantities $\widetilde{A}-\widetilde{G}$. 